\title{
Prospect of Swarm Intelligence (SI) Techniques for the Charging Optimization of Electric Vehicles
}

\author{
Imran Rahman ${ }^{1}$, Pandian M Vasant ${ }^{1 *}$, Balbir Singh Mahinder Singh ${ }^{1}$ and Abdullah-Al-Wadud $\mathbf{M}^{2}$ \\ ${ }^{1}$ Department of Fundamental and Applied Sciences, University Technology PETRONAS, Malaysia \\ ${ }^{2}$ Department of Software Engineering, College of Computer and Information Sciences, King Saud University, Riyadh, Kingdom of Saudi Arabia \\ *Corresponding author: Vasant PM, Department of Fundamental and Applied Sciences, University Technology PETRONAS, Malaysia, Tel: +60 5-368 8000; E-mail: \\ pvasant@gmail.com
}

Received date: Oct 09, 2015; Accepted date: Oct12, 2015; Published date: Oct 14, 2015

Copyright: (c) 2015 Imran Rahman, et al. This is an open-access article distributed under the terms of the Creative Commons Attribution License, which permits unrestricted use, distribution, and reproduction in any medium, provided the original author and source are credited.

\section{Introduction}

The Present Transportation division appearances major renovation towards electrification with the balance of the recently emerged smart grid for achieving sustainable and carbon-free future. Electric Vehicles (EVs) are gaining establishment as an environmentally friendly alternative to traditional vehicle, and playing a vibrant role in enhancing the total fuel efficiency. Alternating renewable energy resources can be combined proficiently to the power grid by the greater involvement of EVs in the highways. Formation of appropriate charging infrastructures along with optimum charging control is necessary for further success in the road transport system. Without proper standardization and infrastructure facilities, large number of EVs charging will burden the existing grid hence instability of power grid. The researchers are trying to develop efficient control mechanism for charging infrastructure in order to facilitate upcoming PHEVs penetration in highways. Intelligent energy management is an important issue that has already drawn much attention of researchers. Most of these works require formulation of mathematical models with extensive use of computational intelligence-based optimization techniques to solve many technical problems. This short note deals with the future prospect of Swarm Intelligence (SI) for the Charging optimization of EVs.

Charging of EVs influences many parameters such as power rating, time of charging and location, cost, charging equipment, and effect on the power grid. Issues like charging time, distribution, standardization of demand policies for charging stations and proper regulatory procedures are needed to be addressed for the successful deployment of Electric vehicle charging infrastructure [1].

Swarm intelligence came from the mimic of the living colony such as ant, bird, and fish in nature, which shows unparalleled excellence in swarm than in single in food seeking or nest building [2]. Drawing inspiration from this, researches design many algorithms simulating colony living, such as ant colony algorithm (ACO), particle swarm optimization (PSO) algorithm, artificial bee colony (ABC) algorithm, and gravitational search algorithm (GSA), which shows excellent performance in dealing with complex optimization problems.

Most of the Real-world EVs optimization problems are naturally non-linear, multi-modal with uncertainty and require computationally intensive techniques [3]. SI techniques have been an exciting substitute for giving reasonable solutions. The individual characteristics of the continuous swarm algorithms are very useful for these applications. However, to apply them to a particular optimization problem requires parameter tuning and variable initialization [4].

Hybrid SI techniques are the latest addition in the field of optimization. In order to utilize the benefits of two or more single SI techniques, researchers are trying to combine them in order to get better results in terms of computation time and best fitness value. With the increasing progress of SI research arena, a large amount of techniques are proposed one after another. The advantages and the shortcomings of a particular swarm intelligence technique becomes clear to be seen when the number of its application increases. To overcome the shortcomings, some hybrid methods are proposed by the researchers. It is almost difficult to discover an algorithm that can cope up with all situations and get excellent results in EVs applications like unit commitment, state-of-charge optimization, charging coordination, energy resource scheduling, etc. Thus, the hybrid SI algorithms are proposed to improve the prevailing algorithms.

Although SI-based methods have established their capability to explore large search spaces, they are comparatively incompetent in fine-tuning the solution. This weakness is usually avoided by means of local search method that is applied to the individuals of the population.

Overall, the knowledge generated from swarm intelligence optimization over the last decade has now become mature. So, the researchers from the optimization domain should come forward to apply SI techniques for the improvement of various parameters related to charging of EVs.

\section{References}

1. Rahman I, Vasant PM, Singh BSM, Abdullah-Al-Wadud M (2014) Optimisation of PHEV/EV charging infrastructures: a review. International Journal of Energy Technology and Policy 10: 280-296.

2. Tsai PW, Chen CW (2014) Review on Swarm Intelligence for Optimization. Computing Science and Technology International Journal 2: 13-17.

3. Rahman I, Vasant P, Singh BSM, Abdullah-Al-Wadud M (2015) Swarm Intelligence-Based Optimization for PHEV Charging Stations. Handbook of Research on Swarm Intelligence in Engineering 374.

4. Vasant P (2015) A Short Note on Novel, Original and Innovative Research Results and Findings: Hybrid Optimization Techniques in Manufacturing Environment. Int J Swarm Intel Evol Comput 4: 116. 